\title{
Oncoplastic partial breast reconstruction: concepts and techniques
}

\author{
Carrie K. Chu ${ }^{1}$, Summer E. Hanson ${ }^{1}$, Rosa F. Hwang ${ }^{2}$, Liza C. $\mathbf{W u}^{3}$ \\ ${ }^{1}$ Department of Plastic Surgery, ${ }^{2}$ Department of Breast Surgical Oncology, Division of Surgery, The University of Texas MD Anderson Cancer \\ Center, Houston, TX, USA; ${ }^{3}$ Section of Plast Reconstr Surg, Department of Surgery, The University of Pennsylvania Health System, Philadelphia, \\ PA, USA \\ Contributions: (I) Conception and design: All authors; (II) Administrative support: All authors; (III) Provision of study materials or patients: All \\ authors; (IV) Collection and assembly of data: All authors; (V) Data analysis and interpretation: All authors; (VI) Manuscript writing: All authors; (VII) \\ Final approval of manuscript: All authors. \\ Correspondence to: Carrie K. Chu, MD, MS, FACS. Assistant Professor, Department of Plastic Surgery, University of Texas MD Anderson Cancer \\ Center, 1400 Pressler St, Unit 1488, Houston, TX, USA. Email: CKChu@mdanderson.org.
}

\begin{abstract}
Immediate oncoplastic breast reconstruction performed at the time of breast conserving surgery for the treatment of breast cancer merges the therapeutic goals of complete oncologic extirpation with preservation of breast form and function. A constellation of surgical techniques that employs breast volume displacement and/or replacement methods of varying complexity levels have emerged, thus broadening the potential applications for breast conservation therapy to include cases with increased tumor-to-native breastvolume ratios, multicentric or multifocal disease, and/or previous margin-positive resections. This review describes the various reconstructive methods, including the use of local tissue rearrangement, oncoplastic reduction-mastopexy, and locoregional flaps. Classification of the surgical options into levels I and II volumedisplacement and volume-replacing techniques is made. Additionally, we explore the oncologic safety and effectiveness of this treatment paradigm by summarizing existing supportive evidence regarding associated risk of surgical complications, rate of margin-positive resection, implications for radiographic surveillance, local recurrence rates, and patient-reported outcomes. In conclusion, surgeons may use a wide variety of oncoplastic techniques for partial breast reconstruction at the time of segmental mastectomy to deliver effective breast conserving treatment for women with breast cancer. A growing body of literature affirms the oncologic safety of this approach. Future directions for research include long-term follow-up data with emphasis on outcomes from patient perspectives.
\end{abstract}

Keywords: Breast conservation; breast cancer; breast reconstruction; segmental mastectomy; lumpectomy

Submitted Mar 21, 2020. Accepted for publication Jul 13, 2020.

doi: $10.21037 /$ gs-20-380

View this article at: http://dx.doi.org/10.21037/gs-20-380

\section{Rationale \& goals of oncoplastic breast reconstruction}

Of the approximately 250,000 new cases of breast cancer diagnosed in the United States each year, the majority present with localized disease that is confined to the primary site (1). Over the past decades, numerous observational and randomized controlled trials have demonstrated the equivalent survival conferred by breast-conserving surgery
(BCS) with adjuvant radiation compared with mastectomy for patients with early-stage breast cancer (2-7). With the added benefits of less invasive and shorter surgeries, faster recovery, less frequent complications, preservation of sensation, and psychosocial advantages, breast conservation therapy (BCT) has thus evolved to become a nationallyaccepted standard of care treatment for this patient population (8). 
The goals of BCT include the complete, margin-negative, localized removal of cancer in conjunction with adjuvant radiation to achieve equivalent survival relative to total mastectomy while preserving satisfactory breast form and function. Small tumor-to-breast-volume ratios and favorable tumor locations represent critical features that facilitate satisfactory cosmetic results. Depending upon the definition used, between $20 \%$ to $30 \%$ of patients who undergo traditional BCT suffer unacceptable deformities (9). These are typically characterized by volume asymmetry, skin deficiency, contour deformities, and/or nipple-areolar complex (NAC) malposition (10). Secondary correction of these defects may be challenging due to radiation-associated fibrosis and vascular injury (10).

The emergence of oncoplastic breast reconstruction performed immediately at the time of segmental mastectomy or prior to initiation of radiotherapy represents a powerful approach that aims to preemptively mitigate these undesirable sequelae by either remolding the breast tissue remnant into a favorable shape and size or replacement of the excision volume with locoregional tissue $(11,12)$. At the same time, the oncologic principles of BCT should remain uncompromised; these include margin-negative local tumor clearance and avoidance of complications that compromise timely delivery of adjuvant therapies. In this manner, oncoplastic breast reconstruction offers the potential to expand applications of BCS beyond its traditional indications, to include patients with large tumor-to-breast-volume ratios, multicentric or multifocal disease, diffuse microcalcifications, and previously positive segmental resection margins (13-15).

The American Society of Breast Surgeons defines oncoplastic breast reconstruction as "a form of breastconservation surgery that includes oncologic resection with a partial mastectomy, ipsilateral reconstruction using volume displacement or volume replacement techniques with possible contralateral symmetry surgery when appropriate" (11). This review provides a comprehensive discussion of preoperative considerations, technical procedures, and outcomes to support the safety and effectiveness associated with immediate oncoplastic breast reconstruction as part of BCT for the treatment of patients with breast cancer.

\section{Preoperative considerations}

Preoperative assessment to determine the suitability of oncoplastic reconstruction and BCT should take into consideration a number of patient- and tumor-related variables. Estimated size, focality, and location of the primary lesion and any associated radiographic abnormality should be understood, along with tumor proximity to the nipple areolar complex. The upper inner quadrant is typically less forgiving cosmetically, and reconstruction of defects in this position may require more extensive rearrangement (16). Central, subareolar tumors occasionally require sacrifice of the NAC; even with oncologic preservation, the resultant resection cavity often undermines the NAC and therefore limits the vascularity to support safe NAC repositioning. For tumors with close proximity to the skin, any possibility of oncologic or ischemic skin loss should be anticipated. The tumor-to-breast-volume ratio should be evaluated within the context of patient expectations regarding desired breast size to determine feasibility and approach.

Baseline comorbidities that may compromise wound healing, including smoking, steroid use, diabetes, and morbid obesity, should be noted in order to minimize subsequent delayed healing and potential interference with adjuvant treatment. Avoidance of surgical maneuvers involving extensive undermining, thinned skin flaps, excessive tension, triple-point closure, and substantial NAC repositioning may be desirable. Examination should note breast size, skin quality, ptosis, previous scars, and asymmetry. Coordinated incision planning with the ablative surgeon will optimize both oncologic and cosmetic outcomes.

\section{Techniques of oncoplastic breast reconstruction}

\section{Volume displacement techniques}

Volume displacement techniques make use of the remnant breast tissue after segmental resection to restore aesthetic breast forms $(17,18)$. Reduction in the size of the overall breast is requisite to varying degrees, and the amount of remaining native breast tissue inherently limits the size of the eventual reconstructed breast. Patient expectations should be adequately addressed. Recent efforts to standardize the definitions of oncoplastic volume displacement procedures have resulted in classification based on the amount of native tissue removed during segmental mastectomy $(11,19)$.

\section{Level 1 volume displacement procedures}

Reconstruction of defects that result from removal of less than $20 \%$ of the native breast tissue in women with small to 

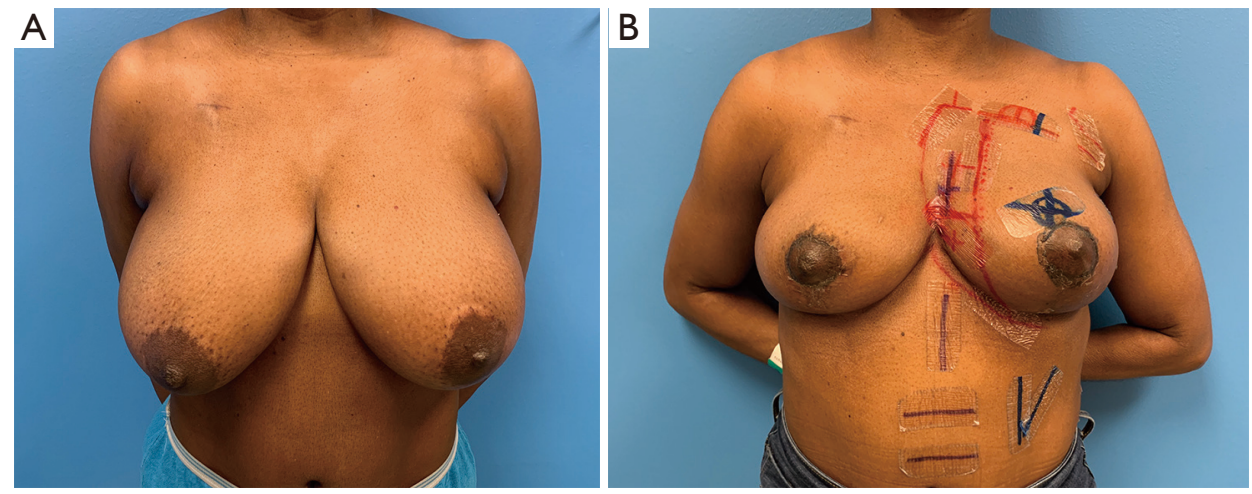

Figure 1 Wise pattern mastopexy-reduction techniques may be used to for patients with ptosis, macromastia, and skin excess. (A) Preoperative photo of 45-year-old female with left T3N1 breast cancer and bilateral ptosis who presents for breast conservation therapy. (B) Postoperative photo at four weeks after patient underwent segmental mastectomy with bilateral immediate superomedial pedicle Wise pattern oncoplastic mastopexy. Radiation markers are in place.

moderate breast size are typically performed using Level 1 volume displacement procedures (11). Small tumors arising from the background of ample native breast tissue are amenable to limited rearrangement of local parenchymal tissue to fill the resultant cavity. These techniques typically involve mobilization of leading edges of the cavity walls by some degree of subcutaneous undermining and elevation from the pectoralis fascia with or without back cuts (20). The parenchymal flaps are then sutured into position. Care should be taken to avoid tethering or distortion of the NAC.

A variety of incision locations may provide suitable access for concomitant segmental resection and adjacent tissue transfer. Periareolar incisions that encompass varying lengths along the NAC circumference are common. These incisions may be modified with small lateral radial extensions, lateral wedge skin excisions, and/or superior crescentic skin excisions to achieve limited NAC suspension. Crescent and doughnut mastopexies both fall into this category (20).

For tumors that are more peripherally-located relative to the NAC, periareolar access may require excessive undermining to reach the oncologic site. In these cases, curvilinear incisions that parallel the curve of the NAC may be placed directly overlying the site of concern. Inframammary fold incisions may be used to approach lower pole tumors. Finally, the axillary sentinel node incision may provide access to upper outer quadrant lesions. In general, incisions over the upper inner quadrant should be avoided if possible to limit visibility.

For larger parenchymal defects, application of the round block technique may be considered $(21,22)$. This approach makes use of two concentric circular incisions around the NAC followed by subcutaneous undermining that may be extended throughout the breast envelope circumferentially to maximize access if necessary. After appropriate excision, parenchymal flaps are then developed using partial undermining over the pectoralis fascia and used to collapse the resultant cavity. The NAC remains attached to the central parenchymal mound and thus remains vascularized.

\section{Level 2 volume displacement procedures}

Reconstruction of defects that result from removal of between $20 \%$ to $50 \%$ of the native breast tissue may be addressed using Level 2 volume displacement procedures, especially in women with moderate to large size breasts (11). For patients with macromastia or ptosis, application of reduction-mastopexy techniques in the oncoplastic setting offers potent tools to address larger resection defects while concomitantly improving breast shape and size $(23,24)$. The substantial parenchymal rearrangement allows for complete defect obliteration while removing skin excess and repositioning of the NAC into a more favorable location. With proper understanding of anatomic breast aesthetics and tissue perfusion, a number of combinations of skin excision patterns and dermatoglandular pedicles to support NAC vascularity may be exercised in order to address tumors in all breast quadrants.

The Wise skin excision pattern is a popular technique that provides skin reduction of the dissociated skin envelope and remolding of the internal parenchyma with elevation of the NAC on the final breast mound $(23,25,26)$ (Figure 1). 

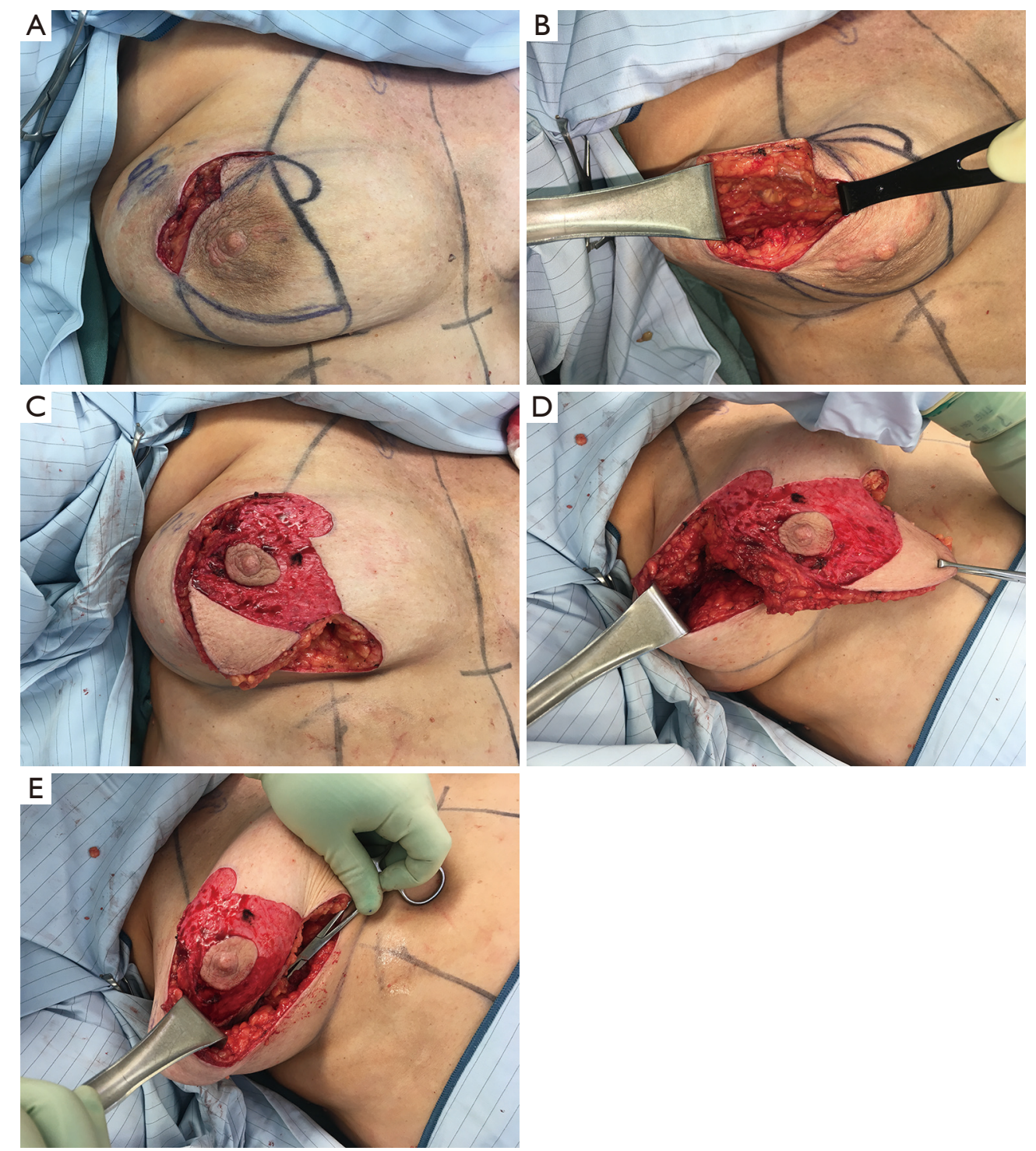

Figure 2 For patients with ptosis without significant macromastia or skin excess, circumvertical mastopexy techniques may be useful. Intraoperative photos of a 47-year-old female with right upper outer quadrant breast cancer undergoing segmental mastectomy via coordinated incisional approach. A circumvertical superomedial mastopexy was performed with preservation of the inferior pole wedge, which was rotated with the pedicle for defect obliteration.

The pattern may be combined with any number of dermatoglandular pedicle designs that both supply the NAC and fill the defect. Inferior, superomedial, medial, central mound, and lateral pedicles represent possible options, selection of which should be dictated primarily by tumor location and secondarily by baseline breast dimensions. Closure of the Wise pattern incisions results in an inverted "T" configuration; care should be taken to avoid excessive tension at the three-point " $\mathrm{T}$ "-junction as this site is susceptible to ischemia and delayed healing.

When skin excess and ptosis severity are limited, circumvertical short scar mastopexy techniques avoid the creation of a T-junction $(17,23)$ (Figure 2). This method creates breast reshaping using vertical parenchymal pillars that provide durable support of the glandular mound without relying on the elastic outer skin envelope to provide the aesthetic breast shape. The circumvertical mastopexy pattern typically relies on a superomedial, superior, or 
medial pedicle. Tumors located in these positions require cautious pedicle design. Durability, short scar length, and improved wound healing represent potential advantages over the more traditional Wise pattern technique.

Patients who smoke or otherwise carry risk factors for adverse post-surgical healing such as diabetes mellitus, steroid use, etc., should be carefully screened when considering oncoplastic reduction-mastopexy. Necrosis of the NAC is a rare but material complication. Delayed healing at the T-junction requires diligent observation and prompt intervention if indicated to avoid delay of adjuvant treatments. The timing of contralateral symmetry reduction-mastopexy procedures that are typically necessary should be discussed preoperatively. Performing these procedures concomitantly with the oncoplastic reconstruction can obviate the need for secondary surgical procedures, but requires estimation of post-radiation changes of the affected breast to optimize eventual symmetry.

\section{Volume replacement techniques}

The ability to replace the resected parenchymal volume using the addition of vascularized locoregional tissue further augments the range of potential cases amenable to BCT (27-31). While some of these flaps rely upon randompattern blood supply, contemporary familiarity with perforator dissection techniques has facilitated a number of axial-pattern and islandized locoregional flaps within the posterolateral thorax that optimize flap reach, inset, vascularity, and donor site morbidity. These flaps also offer the possibility for replacement of cutaneous defects.

\section{Thoracoepigastric flap}

The adipocutaneous tissue within the thoracoepigastric region immediately below the inframammary fold can provide tissue replacement for lower pole resection defects $(31,32)$. The thoracoepigastric flap may be based anteriorly or posteriorly, and its width is limited by the tissue laxity to allow for primary closure along the inframammary fold. The anteriorly-based flap is supported by the anterior intercostal artery perforators, while the lateral intercostal and lateral thoracic artery branches sustain the posteriorly-based flap. Flap inset may be limited by the typical preservation of the proximal skin bridge, and the inframammary fold position is displaced inferiorly.

\section{Lateral intercostal artery perforator flap}

The lateral intercostal artery perforators originating from the fifth, sixth, or seventh interspaces may sustain robust blood supply to this flap $(31,33)$. The vessels arise $2-3 \mathrm{~cm}$ posterior to the lateral border of the pectoralis. The associated angiosome corresponds well with the transversely-oriented adipocutaneous excess over the lateral chest which is often present adjacent to the lateral breast border. Once the perforator is identified and fully dissected, the flap is islandized, thus generating great degrees of freedom of motion that facilitate transposition into the defect via a developed tunnel. Lateral to central defects are suitable for this application (34) (Figure 3).

\section{Lateral thoracic artery perforator flap}

The lateral thoracic artery originates from the axillary artery and descends along the lateral border of the pectoralis muscle. Muscular and terminal branches supply the pectoralis and serratus anterior. Cutaneous branches also arise at the third and fourth interspaces, slightly further cephalad and anterior to the position of the lateral intercostal artery perforators. Dissected fully proximally, the pedicle provides a wide arc of rotation to reach central and lateral defects (35).

\section{Thoracodorsal artery perforator and latissimus flaps} A branch of the subscapular artery, the thoracodorsal artery supplies the latissimus dorsi muscle via an anterior descending branch and a posterior transverse branch coursing along the deep surface of the muscle (36). From the anterior branch beginning at approximately 6 to $8 \mathrm{~cm}$ from the vessel origin, a number of musculocutaneous perforators arise upon which fasciocutaneous flaps may be based (36). Alternatively, a partial muscle-sparing pedicled flap may be designed centered on the anterior branch. These variations relying upon the thoracodorsal pedicle provide additional locoregional options for partial breast reconstruction (37).

Traditionally, use of the total latissimus muscle or myocutaneous flap for partial breast reconstruction after BCS has been reserved for correction of secondary postradiation deformities. However, immediate latissimus flap reconstruction for large-volume defects after partial mastectomy has also been described (38). Use of flaps based on the thoracodorsal vascular system in the setting of BCT should be carefully weighed against the potential need for use of the latissimus flap for whole breast reconstruction in the event of subsequent local recurrence and/or need for mastectomy.

In summary, locoregional flaps of the posterolateral 

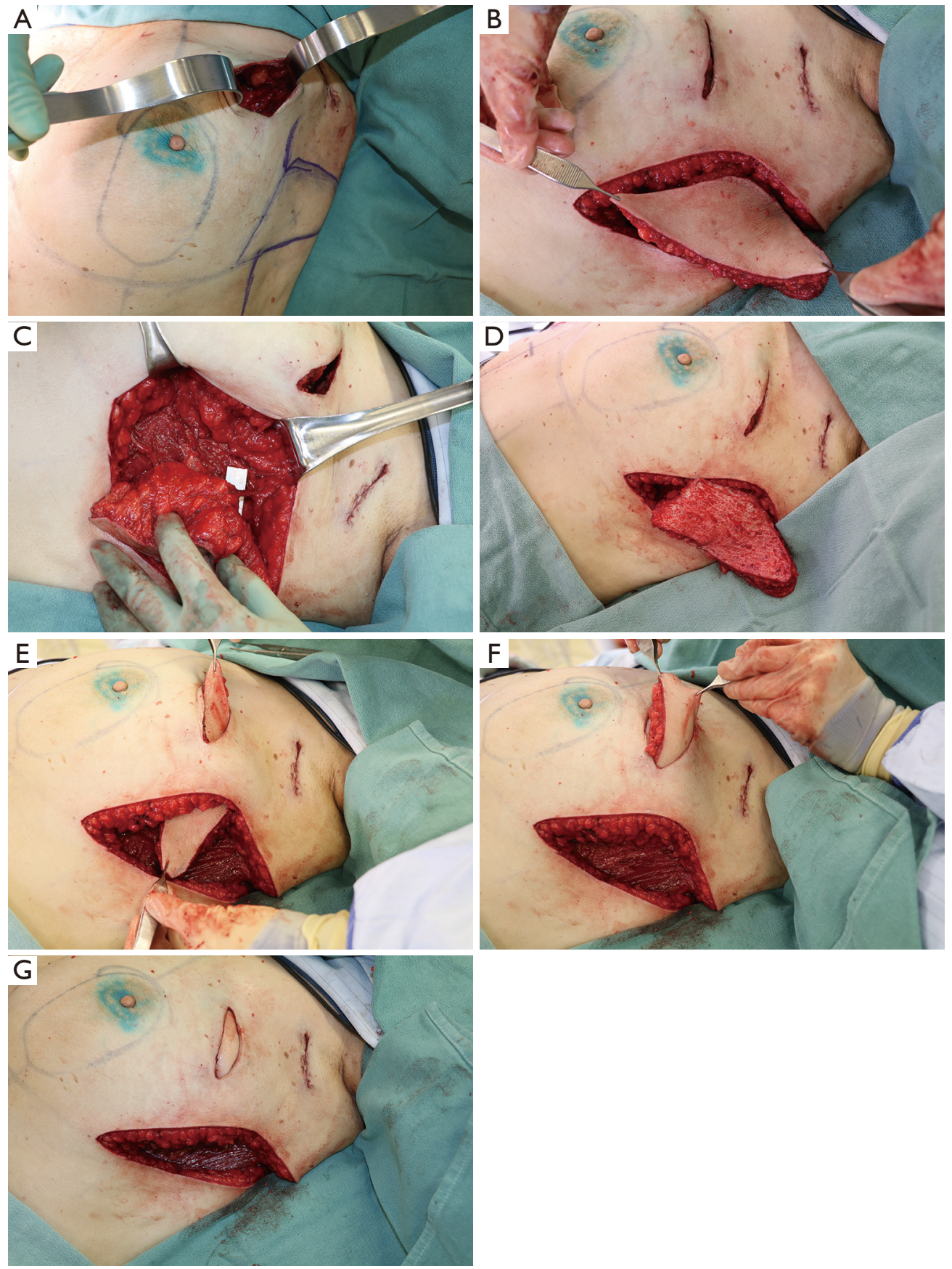

Figure 3 For patients without indication for mastopexy or in whom mastopexy is contraindicated, central sub-areolar tumors, or large tumor-to-breast size ratios, use of a pedicled locoregional flap is an alternative. Intraoperative photos of a 53-year-old female with left upper outer pole breast cancer who underwent segmental mastectomy and immediate tissue replacement with a lateral intercostal artery perforator flap based off of the fifth intercostal vascular pedicle. The flap was de-epithelialized, islanded, and transposed for defect obliteration. 
thorax may be considered in the following scenarios:

(I) High ratio of tumor-to-native breast size;

(II) Outer quadrant tumors;

(III) Central tumors not amenable to oncoplastic mastopexy techniques;

(IV) Anticipated skin excision.

\section{Complications \& delay of adjuvant therapy}

Although oncoplastic reconstruction encompasses a heterogenous collection of techniques, to varying extents, the addition of immediate reconstruction procedures at the time of segmental mastectomy introduces the potential of added complications. Satisfactory achievement of the therapeutic goals of BCT mandates avoidance of complications associated with immediate oncoplastic reconstruction that may interfere with timely initiation of adjuvant therapies.

While randomized controlled studies are lacking, retrospective cohort analyses comparing BCS alone with BCS with immediate oncoplastic reconstruction have generally demonstrated acceptable morbidity profiles $(39,40)$. Reported overall complication rates following oncoplastic surgery range from $8.9 \%$ to $24.6 \%$ depending upon definitions; these estimates compare favorably with historical values associated with BCS alone (39). In one of the largest published single-institution series of immediate oncoplastic reconstruction to-date, including 2,258 cases of BCS alone and 939 cases of BCS with immediate reconstruction, Carter et al. observed significantly higher risk of seroma formation after BCS-only treatment $(18 \%$ versus $13.4 \%, \mathrm{P}=0.0016$ ), while $\mathrm{BCS}$ with reconstruction was associated with greater likelihood of delayed wound healing $(4.8 \%$ versus $1.4 \%, \mathrm{P}<0.001)$. Infection rates were equivalent between the two cohorts $(4.1 \%$ without reconstruction versus $4.4 \%$ with reconstruction, $\mathrm{P}=0.61$ ). Despite the slight increase in wound-related complications, the majority of such cases were mild with few requiring reoperative intervention or delay of adjuvant therapies (41).

Khan et al. evaluated 169 breast cancer patients who required adjuvant chemotherapy following BCS alone, BCS with oncoplastic reconstruction, mastectomy alone, or mastectomy with reconstruction. No significant difference in the median number of days to initiation of chemotherapy was observed (BCS alone 29.5 days, BCS with oncoplastic reconstruction 29 days, mastectomy alone 29 days, mastectomy with reconstruction 31 days) (42). Kelemen et al. reported a single-center retrospective experience involving 378 patients who received oncoplastic breast reconstruction and 378 randomly selected patients who underwent conventional BCS without oncoplastic reconstruction within the same study period. In the oncoplastic group, the minor and major complication rates were $3.1 \%$ and $2.6 \%$, respectively. Amongst the conventional BCS group, minor and major complication rates were $3.1 \%$ and $3.4 \%$, respectively. Median time to initiation of adjuvant treatment was 4.2 weeks for the oncoplastic group and 4.1 weeks in the conventional BCS cohort. These results were all statistically similar (43).

In a meta-analysis by Losken $e t$ al. including ten studies and 1,773 oncoplastic reductions, 1,392 oncoplastic flap reconstructions, and 5,494 BCS-alone patients, the pooled complication rates were $16 \%$ for reduction, $14 \%$ for flap reconstruction, and $25.9 \%$ in the BCS-alone patients. The authors indicate that there were no reported cases of delay in adjuvant therapies as a result of postoperative complications (39). However, a more recent study from the same group reported their institutional experience with 118 cases of oncoplastic reduction, of which $22 \%$ experienced complications including cellulitis, delayed healing, seroma, and dehiscence. The median time to radiation was significantly higher amongst patients who had complications ( 74 versus 54 days, $\mathrm{P}<0.001$ ) (44).

Variable definitions in acceptable intervals to initiation of adjuvant treatment after BCS cloud meaningful interpretation of scant cases of delay reported in the literature $(45,46)$. Even if overall likelihood is low, every effort should be made to avoid wound healing morbidity and consequent treatment delays by using appropriate patient selection and technical precaution.

\section{Margins}

Margin clearance is a critical component of effective BCT, as higher local recurrence rates are associated with positive margins after BCS (47). Numerous studies have examined the issue of resection margins as related to oncoplastic breast reconstruction. A systematic review by De La Cruz et al. found eleven studies including specific margin information on 1,455 patients, of whom $9.8 \%$ were reported as having positive margins. However, according to recently updated guidelines from the American Society of Breast Surgeons and the Society of Surgical Oncology, positive margins are defined as ink on tumor for invasive breast cancer. For ductal carcinoma in-situ, margins less than $2 \mathrm{~mm}$ are considered positive. Using the modified definitions, the 
rate of margin positivity was $7.8 \%$ (40). These estimates compare favorably with historical positive margin rates of $15-47 \%$ in standard lumpectomy (40).

Use of oncoplastic techniques in conjunction with BCS has apparent benefits in improved margin clearance compared with BCS alone by removal of larger volumes of breast tissue. In the aforementioned study by Carter et al. at MD Anderson, the positive margin rate was $5.8 \%$ for oncoplastic reconstruction as opposed to $8.3 \%$ for BCS alone ( $\mathrm{P}=0.04)$ (41). Losken et al. found that the oncoplastic approach was associated with wider negative margins compared with standard BCS (4.3 versus $2.8 \mathrm{~mm}$, $\mathrm{P}=0.01)$. Further, re-excisions were less frequent with use of oncoplastic reconstruction (12\% versus $25.9 \%, \mathrm{P}=0.01$ ) (48). Across 55 studies and 6,011 patients treated with oncoplastic reconstruction, De La Cruz et al. calculated a weighted mean re-excision rate of $6 \%$, which also compares favorably with historical data associated with standard BCS (40,49,50). In Losken et al.'s meta-analysis, benefits in improved margin clearance using oncoplastic reconstruction translated into significantly lower re-excision rates $(2.94 \%$ reduction, 5.66\% local flaps, $14.6 \%$ BCS only, $\mathrm{P}<0.001)$. However, oncoplastic reduction was associated with increased likelihood for conversion to mastectomy based on positive margins $(7.87 \%$ reduction, $4.46 \%$ local flaps, $3.79 \%, \mathrm{P}<0.001$ ) (39). This finding may reflect perceived challenges in re-orientation to facilitate re-excision after oncoplastic reduction. Alternatively, larger excision volumes typically associated with oncoplastic reduction may preclude further attempts at breast conservation with persistently positive margins. Predictors of positive margins following oncoplastic breast reconstruction include higher-grade tumors, invasive lobular carcinoma, larger tumor size, and tumor stage (51).

\section{Surveillance}

A potential concern regarding the safety of oncoplastic breast surgery is disruption of normal residual breast parenchyma that may in turn lead to radiographic abnormalities, interfering with reliable future surveillance in breast cancer patients. In a study of postoperative mammograms over 5 years in 17 patients who underwent oncoplastic reduction and 17 patients who completed segmental mastectomy only, Losken et al. observed a trend towards longer time to mammographic stabilization amongst the reduction cohort (25.6 versus 21.2 months). Similarly, trends toward greater numbers of postoperative mammograms and ultrasounds were demonstrated. In turn, the rate of tissue sampling in the oncoplastic group was significantly higher than in the segmental only group $(53 \%$ versus $18 \%$ ) (52). These findings are corroborated by a study by Dolan et al. that shows significantly more frequent ultrasounds and biopsies after oncoplastic reconstruction compared with BCS alone (53).

A larger study by Piper et al. offers contrasting results. In an age-matched analysis of 49 cases of oncoplastic reduction and 49 cases of segmental resection alone, review of mammography reports at 6 months, 1 year, 2 years, and 5 years after surgery showed that most patients had benign postoperative changes such as fat necrosis and calcifications, but radiologists were able to discern between these and more concerning abnormal mammographic findings as evidenced by equivalent rates of total and positive biopsies in both treatment groups (total biopsy rate $18 \%$ segmental mastectomy versus $24 \%$ oncoplastic reduction). At 6 months, no difference in abnormal findings between the oncoplastic and the segmental-mastectomy-only groups were found. At 1 year, the oncoplastic cohort exhibited significantly more abnormal findings for which biopsy was recommended. By the 2- and 5-year timepoints, however, the difference in rate of abnormal findings had subsided. Benign calcifications were more common in the reduction group at 1,2 , and 5 years (54).

It is apparent from these studies that postoperative changes may prompt attention after BCS with or without substantial tissue arrangements; with the passage of time, stabilization typically occurs. The threshold to perform further workup and/or tissue sampling likely varies by institution, but multi-disciplinary communication and coordination during surveillance will optimize management strategies should concern for radiographic abnormalities arise.

\section{Local recurrence}

The reported range of local recurrence following oncoplastic reconstruction within the BCT paradigm is $2-6.8 \%$ (39). The addition of oncoplastic reconstruction to $\mathrm{BCS}$ is not associated with higher risk of local recurrence compared with conventional BCS. The study by Carter et al. showed that, adjusted for age, nodal stage, grade, margin status, lymphovascular invasion, hormone receptor status, and adjuvant radiation, BCS with oncoplastic reconstruction was associated with equivalent recurrence-free survival compared with BCS alone. Three-year recurrence- 
free survival was likewise statistically similar (BCS with oncoplastic $94.6 \%$ versus BCS only 96.1\%) (41). Multiple additional studies corroborate these findings $(43,55,56)$.

What remains unclear, however, is whether the wider margin benefits attributed to the oncoplastic approach has the potential to translate into lower local recurrence rates than traditional BCS when combined with appropriate adjuvant therapies. To date, there is no definitive data to support this theoretical advantage. In an analysis of 980 patients, of whom 104 underwent oncoplastic breast conserving surgery, 558 conventional BCS, and 318 mastectomy with immediate reconstruction, five-year local recurrence rates were statistically equivalent amongst all three groups (oncoplastic 2\%, BCS only 3.4\%, mastectomy $2.6 \%, \mathrm{P}=0.973$ ) (57). A meta-analysis including 13 studies and 15,883 patients (5176 oncoplastic, 10,707 control including standard BCS or mastectomy) demonstrated no significant difference between treatment with oncoplastic surgery and standard BCS/mastectomy (risk ratio 0.861 , 95\% CI: 0.64-1.16, $\mathrm{P}=0.296)(58)$.

\section{Patient-reported outcomes}

Contemporary evaluation of the effectiveness of healthcare interventions emphasizes the importance of patientreported outcomes after treatment. For patients who have undergone $\mathrm{BCT}$, a dedicated module of the validated BREAST-Q measurement tool is available for assessment of domains in satisfaction with breasts, psychosocial wellbeing, sexual well-being, and physical well-being $(59,60)$. As the oncologic safety of these procedures continue to be affirmed, assessment of the effectiveness of oncoplastic breast reconstruction in the context of BCT using patientreported outcomes measurement tools represents the next research frontier to further validate the use of these procedures.

Researchers have adopted varying perspectives to meaningful comparison and assessment of quality of life after oncoplastic breast reconstruction. Losken et al. performed a prospective analysis examining preoperative and postoperative quality of life as measured by the BREAST-Q in 353 patients who underwent BCS and oncoplastic reduction mammaplasty. Over one year postoperatively, patient reported significant increase in selfconfidence, feelings of attractiveness and emotional health compared with preoperative baseline, indicating evidence of sustained quality of life benefits associated with this treatment paradigm. However, no comparison to alternative cancer treatment strategies was available (24). Such observed quality of life improvements may be attributable to the benefits of reduction mammaplasty performed in patients with macromastia, but this theoretical advantage is not definitively proven. Di Miccio et al. compared 87 cases of BCS alone with 32 cases of BCS with bilateral reduction in patients with macromastia and early stage breast cancer. Although no statistically-significant benefit with reduction mammaplasty was found, there was a trend towards improved satisfaction with the breast amongst the reconstruction cohort (61).

Ojala et al. surveyed 293 patients who underwent BCS alone and 86 who received oncoplastic treatment using the Breast Cancer Treatment Outcome Scale for assessment of aesthetic outcome. Patients in the oncoplastic cohort had significantly larger cancers, greater likelihood of multifocal tumors, bigger resection specimens, and frequency of node-positive disease. Aesthetic results were better in the BCS-only patients, likely reflecting the more aggressive disease profile in the oncoplastic group. After adjustment, multifocality remained a predictor of poor aesthetic outcome for patients with BCS alone, but was not associated with adverse aesthetic result in the oncoplastic cohort (62).

Kelemen et al. evaluated 350 cases of oncoplastic reconstruction and 350 cases of conventional BCS using the European Organisation for Research and Treatment of Cancer- Quality of Life Questionnaire. At one year after surgery, patients treated with oncoplastic reconstruction reported significantly better quality of life outcomes including emotional and social functioning and body image compared with those following conventional BCS (43).

Using the Danish Breast Cancer Cooperative Group registry, Rose et al. compared quality of life outcomes as measured by the BREAST-Q BCT postoperative module amongst a cohort of 200 patients treated with oncoplastic breast reconstruction 1,304 patients with BCS alone. Oncoplastic treatment was associated with a significantly improved psychosocial well-being [odds ratio (OR) 2.15, 95\% confidence interval (CI): 1.25-3.69] compared with BCS only. While both satisfaction with breast (OR 0.95 , 95\% CI: $0.57-1.59$ ) and sexual wellbeing (OR 1.42, 95\% CI: $0.78-2.58)$ were similar in both groups, the addition of oncoplastic surgery was not associated with more physical discomfort as measured by reported physical well-being (OR 0.83, 95\% CI: 0.50-1.39) (63).

BCT has been shown to confer significant long-term quality of life advantages over mastectomy $(64,65)$. While emerging evidence appears to support benefits associated 
with the addition of oncoplastic reconstruction to the BCT paradigm, direct comparison of patient-reported outcomes using this approach with results following mastectomy is limited. Kelsall et al. evaluated 286 patients with oncoplastic reconstruction and 281 patients with mastectomy and immediate reconstruction. Patients were matched for age, tumor size, and date of surgery. Overall, oncoplastic reconstruction was associated better body image score, self-rated breast appearance, greater return to work and improved function. Once stratified by breast size, case-matched women with larger breasts treated by the oncoplastic method reported better body image and self-rated breast appearance scores compared with their mastectomy counterparts. No significant difference was observed for women with smaller breasts (66). Overall, long-term follow-up studies including patient-reported measures are necessary.

\section{Conclusions}

Surgeons may use a wide variety of oncoplastic techniques for partial breast reconstruction at the time of segmental mastectomy to deliver effective breast conserving treatment for women with breast cancer. A growing body of literature affirms the oncologic safety of this approach. Future directions for research include long-term follow-up data with emphasis on outcomes from patient perspectives.

\section{Acknowledgments}

Funding: None.

\section{Footnote}

Provenance and Peer Review: This article was commissioned by the Guest Editors (Charles E Butler, Carrie Chu, and Margaret Roubaud) for the series "New Frontiers in Breast Reconstruction" published in Gland Surgery. The article was sent for external peer review organized by the Guest Editors and the editorial office.

Conflicts of Interest: All authors have completed the ICMJE uniform disclosure form (available at http://dx.doi. org/10.21037/gs-20-380). The series "New Frontiers in Breast Reconstruction" was commissioned by the editorial office without any funding or sponsorship. Dr. CKC served as the unpaid Guest Editor of the series. The authors have no other conflicts of interest to declare.
Ethical Statement: The authors are accountable for all aspects of the work in ensuring that questions related to the accuracy or integrity of any part of the work are appropriately investigated and resolved.

Open Access Statement: This is an Open Access article distributed in accordance with the Creative Commons Attribution-NonCommercial-NoDerivs 4.0 International License (CC BY-NC-ND 4.0), which permits the noncommercial replication and distribution of the article with the strict proviso that no changes or edits are made and the original work is properly cited (including links to both the formal publication through the relevant DOI and the license). See: https://creativecommons.org/licenses/by-nc-nd/4.0/.

\section{References}

1. National Cancer Institute Surveillance, Epidemiology, and End Results Program. Cancer Stat Facts: Female Breast Cancer. Available online: https://seer.cancer.gov/statfacts/ $\mathrm{html} /$ breast.html

2. Veronesi U, Cascinelli N, Mariani L, et al. Twentyyear follow-up of a randomized study comparing breastconserving surgery with radical mastectomy for early breast cancer. N Engl J Med 2002;347:1227-32.

3. Arriagada R, Lê MG, Rochard F, et al. Conservative treatment versus mastectomy in early breast cancer: patterns of failure with 15 years of follow-up data. Institut Gustave-Roussy Breast Cancer Group. J Clin Oncol 1996;14:1558-64.

4. Forrest AP, Stewart HJ, Everington D, et al. Randomised controlled trial of conservation therapy for breast cancer: 6-year analysis of the Scottish trial. Scottish Cancer Trials Breast Group. Lancet 1996;348:708-13.

5. Jacobson JA, Danforth DN, Cowan KH, et al. Ten-year results of a comparison of conservation with mastectomy in the treatment of stage I and II breast cancer. N Engl J Med 1995;332:907-11.

6. Lichter AS, Lippman ME, Danforth DN Jr, et al. Mastectomy versus breast-conserving therapy in the treatment of stage I and II carcinoma of the breast: a randomized trial at the National Cancer Institute. J Clin Oncol 1992;10:976-83.

7. Poggi MM, Danforth DN, Sciuto LC, et al. Eighteenyear results in the treatment of early breast carcinoma with mastectomy versus breast conservation therapy: the National Cancer Institute Randomized Trial. Cancer 2003;98:697-702. 
8. NIH consensus conference. Treatment of early-stage breast cancer. JAMA 1991;265:391-5.

9. Clough KB, Cuminet J, Fitoussi A, et al. Cosmetic sequelae after conservative treatment for breast cancer: classification and results of surgical correction. Ann Plast Surg 1998;41:471-81.

10. Clough KB, Nos C, Fitoussi A, et al. Partial reconstruction after conservative treatment for breast cancer: classification of sequelae and treatment options. Ann Chir Plast Esthet 2008;53:88-101.

11. Chatterjee A, Gass J, Patel K, et al. A Consensus Definition and Classification System of Oncoplastic Surgery Developed by the American Society of Breast Surgeons. Ann Surg Oncol 2019;26:3436-44.

12. Noguchi M, Yokoi-Noguchi M, Ohno Y, et al. Oncoplastic breast conserving surgery: Volume replacement vs. volume displacement. Eur J Surg Oncol 2016;42:926-34.

13. Crown A, Laskin R, Rocha FG, et al. Extreme oncoplasty: Expanding indications for breast conservation. Am J Surg 2019;217:851-6.

14. Tan MP, Sitoh NY, Sim AS. Breast conservation treatment for multifocal and multicentric breast cancers in women with small-volume breast tissue. ANZ J Surg 2017;87:E5-E10.

15. Losken A, Hart AM, Dutton JW, et al. The Expanded Use of Autoaugmentation Techniques in Oncoplastic Breast Surgery. Plast Reconstr Surg 2018;141:10-9.

16. Kronowitz SJ, Kuerer HM, Buchholz TA, et al. A management algorithm and practical oncoplastic surgical techniques for repairing partial mastectomy defects. Plast Reconstr Surg 2008;122:1631-47.

17. Anderson BO, Masetti R, Silverstein MJ. Oncoplastic approaches to partial mastectomy: an overview of volumedisplacement techniques. Lancet Oncol 2005;6:145-57.

18. Yang JD, Lee JW, Cho YK, et al. Surgical techniques for personalized oncoplastic surgery in breast cancer patients with small- to moderate-sized breasts (part 1): volume displacement. J Breast Cancer 2012;15:1-6.

19. Clough KB, Kaufman GJ, Nos C, et al. Improving breast cancer surgery: a classification and quadrant per quadrant atlas for oncoplastic surgery. Ann Surg Oncol 2010;17:1375-91.

20. Patel K, Bloom J, Nardello S, et al. An Oncoplastic Surgery Primer: Common Indications, Techniques, and Complications in Level 1 and 2 Volume Displacement Oncoplastic Surgery. Ann Surg Oncol 2019;26:3063-70.

21. Burrah R, James K, Lund J, et al. Breast conservation surgery by round block mammoplasty. Eur J Surg Oncol
2020;46:240-4.

22. Refaat M, Abouelnagah G, Awad AT, et al. Modified round block technique for peripherally located early cancer breast, a technique that fits for all quadrants. Breast J 2020;26:414-9.

23. Rose JF, Colen JS, Ellsworth WAt. Reduction and Mastopexy Techniques for Optimal Results in Oncoplastic Breast Reconstruction. Semin Plast Surg 2015;29:102-9.

24. Losken A, Hart AM, Broecker JS, et al. Oncoplastic Breast Reduction Technique and Outcomes: An Evolution over 20 Years. Plast Reconstr Surg 2017;139:824e-33e.

25. Losken A, Styblo TM, Carlson GW, et al. Management algorithm and outcome evaluation of partial mastectomy defects treated using reduction or mastopexy techniques. Ann Plast Surg 2007;59:235-42.

26. Bong J, Parker J, Clapper R, et al. Clinical series of oncoplastic mastopexy to optimize cosmesis of largevolume resections for breast conservation. Ann Surg Oncol 2010;17:3247-51.

27. Ho W, Stallard S, Doughty J, et al. Oncological Outcomes and Complications After Volume Replacement Oncoplastic Breast Conservations-The Glasgow Experience. Breast Cancer (Auckl) 2016;10:223-8.

28. Lee JW, Kim MC, Park HY, et al. Oncoplastic volume replacement techniques according to the excised volume and tumor location in small- to moderate-sized breasts. Gland Surg 2014;3:14-21.

29. Park HC, Kim HY, Kim MC, et al. Partial breast reconstruction using various oncoplastic techniques for centrally located breast cancer. Arch Plast Surg 2014;41:520-8.

30. Yang JD, Kim MC, Lee JW, et al. Usefulness of Oncoplastic Volume Replacement Techniques after Breast Conserving Surgery in Small to Moderate-sized Breasts. Arch Plast Surg 2012;39:489-96.

31. Yang JD, Lee JW, Cho YK, et al. Surgical techniques for personalized oncoplastic surgery in breast cancer patients with small- to moderate-sized breasts (part 2): volume replacement. J Breast Cancer 2012;15:7-14.

32. Losken A, Hamdi M. Partial breast reconstruction: current perspectives. Plast Reconstr Surg 2009;124:722-36.

33. Hamdi M, Spano A, Van Landuyt K, et al. The lateral intercostal artery perforators: anatomical study and clinical application in breast surgery. Plast Reconstr Surg 2008;121:389-96.

34. Kim JB, Kim DK, Lee JW, et al. The usefulness of pedicled perforator flap in partial breast reconstruction after breast conserving surgery in Korean women. Arch 
Plast Surg 2018;45:29-36.

35. McCulley SJ, Schaverien MV, Tan VKM, et al. Lateral thoracic artery perforator (LTAP) flap in partial breast reconstruction. J Plast Reconstr Aesthet Surg 2015;68:686-91.

36. Saint-Cyr M, Nagarkar P, Schaverien M, et al. The pedicled descending branch muscle-sparing latissimus dorsi flap for breast reconstruction. Plast Reconstr Surg 2009;123:13-24.

37. Rainsbury RM. Breast-sparing reconstruction with latissimus dorsi miniflaps. Eur J Surg Oncol 2002;28:891-5.

38. Mericli AF, Szpalski C, Schaverien MV, et al. The Latissimus Dorsi Myocutaneous Flap Is a Safe and Effective Method of Partial Breast Reconstruction in the Setting of Breast-Conserving Therapy. Plast Reconstr Surg 2019;143:927e-35e.

39. Losken A, Dugal CS, Styblo TM, et al. A meta-analysis comparing breast conservation therapy alone to the oncoplastic technique. Ann Plast Surg 2014;72:145-9.

40. De La Cruz L, Blankenship SA, Chatterjee A, et al. Outcomes After Oncoplastic Breast-Conserving Surgery in Breast Cancer Patients: A Systematic Literature Review. Ann Surg Oncol 2016;23:3247-58.

41. Carter SA, Lyons GR, Kuerer HM, et al. Operative and Oncologic Outcomes in 9861 Patients with Operable Breast Cancer: Single-Institution Analysis of Breast Conservation with Oncoplastic Reconstruction. Ann Surg Oncol 2016;23:3190-8.

42. Khan J, Barrett S, Forte C, et al. Oncoplastic breast conservation does not lead to a delay in the commencement of adjuvant chemotherapy in breast cancer patients. Eur J Surg Oncol 2013;39:887-91.

43. Kelemen P, Pukancsik D, Újhelyi M, et al. Comparison of clinicopathologic, cosmetic and quality of life outcomes in 700 oncoplastic and conventional breast-conserving surgery cases: A single-centre retrospective study. Eur J Surg Oncol 2019;45:118-24.

44. Kapadia SM, Reitz A, Hart A, et al. Time to Radiation After Oncoplastic Reduction. Ann Plast Surg 2019;82:15-8.

45. Fitoussi AD, Berry MG, Famà F, et al. Oncoplastic breast surgery for cancer: analysis of 540 consecutive cases [outcomes article]. Plast Reconstr Surg 2010;125:454-62.

46. Clough KB, Lewis JS, Couturaud B, et al. Oncoplastic techniques allow extensive resections for breast-conserving therapy of breast carcinomas. Ann Surg 2003;237:26-34.

47. Bethke KP. Breast conservation: predictors and treatment of local recurrence. Semin Surg Oncol 1996;12:332-8.

48. Losken A, Pinell-White X, Hart AM, et al. The oncoplastic reduction approach to breast conservation therapy: benefits for margin control. Aesthet Surg J 2014:34:1185-91.

49. McCahill LE, Single RM, Aiello Bowles EJ, et al. Variability in reexcision following breast conservation surgery. JAMA 2012;307:467-75.

50. Wilke LG, Czechura T, Wang C, et al. Repeat surgery after breast conservation for the treatment of stage 0 to II breast carcinoma: a report from the National Cancer Data Base, 2004-2010. JAMA Surg 2014;149:1296-305.

51. Clough KB, Gouveia PF, Benyahi D, et al. Positive Margins After Oncoplastic Surgery for Breast Cancer. Ann Surg Oncol 2015;22:4247-53.

52. Losken A, Schaefer TG, Newell M, et al. The impact of partial breast reconstruction using reduction techniques on postoperative cancer surveillance. Plast Reconstr Surg 2009;124:9-17.

53. Dolan R, Patel M, Weiler-Mithoff E, et al. Imaging Results Following Oncoplastic and Standard Breast Conserving Surgery. Breast Care (Basel) 2015;10:325-9.

54. Piper M, Peled AW, Sbitany H, et al. Comparison of Mammographic Findings Following Oncoplastic Mammoplasty and Lumpectomy Without Reconstruction. Ann Surg Oncol 2016;23:65-71.

55. De Lorenzi F, Hubner G, Rotmensz N, et al. Oncological results of oncoplastic breast-conserving surgery: Long term follow-up of a large series at a single institution: A matched-cohort analysis. Eur J Surg Oncol 2016;42:71-7.

56. Borm KJ, Schönknecht C, Nestler A, et al. Outcomes of immediate oncoplastic surgery and adjuvant radiotherapy in breast cancer patients. BMC Cancer 2019;19:907.

57. Mansell J, Weiler-Mithoff E, Stallard S, et al. Oncoplastic breast conservation surgery is oncologically safe when compared to wide local excision and mastectomy. Breast 2017;32:179-85.

58. Kosasih S, Tayeh S, Mokbel K, et al. Is oncoplastic breast conserving surgery oncologically safe? A meta-analysis of 18,103 patients. Am J Surg 2020;220:385-392.

59. O'Connell RL, DiMicco R, Khabra K, et al. Initial experience of the BREAST-Q breast-conserving therapy module. Breast Cancer Res Treat 2016;160:79-89.

60. Stolpner I, Heil J, Feißt M, et al. Clinical Validation of the BREAST-Q Breast-Conserving Therapy Module. Ann Surg Oncol 2019;26:2759-67.

61. Di Micco R, O'Connell RL, Barry PA, et al. Standard wide local excision or bilateral reduction mammoplasty in large- 
breasted women with small tumours: Surgical and patientreported outcomes. Eur J Surg Oncol 2017;43:636-41.

62. Ojala K, Meretoja TJ, Leidenius MHK. Aesthetic and functional outcome after breast conserving surgery Comparison between conventional and oncoplastic resection. Eur J Surg Oncol 2017;43:658-64.

63. Rose M, Svensson H, Handler J, et al. Patient-reported outcome after oncoplastic breast surgery compared with conventional breast-conserving surgery in breast cancer. Breast Cancer Res Treat 2020;180:247-56.

64. Lagendijk M, van Egdom LSE, van Veen FEE, et al.

Cite this article as: Chu CK, Hanson SE, Hwang RF, Wu LC. Oncoplastic partial breast reconstruction: concepts and techniques. Gland Surg 2021;10(1):398-410. doi: 10.21037/gs-20380
Patient-Reported Outcome Measures May Add Value in Breast Cancer Surgery. Ann Surg Oncol 2018;25:3563-71.

65. Arndt V, Stegmaier C, Ziegler H, et al. Quality of life over 5 years in women with breast cancer after breastconserving therapy versus mastectomy: a population-based study. J Cancer Res Clin Oncol 2008;134:1311-8.

66. Kelsall JE, McCulley SJ, Brock L, et al. Comparing oncoplastic breast conserving surgery with mastectomy and immediate breast reconstruction: Case-matched patient reported outcomes. J Plast Reconstr Aesthet Surg 2017;70:1377-85. 